\title{
Impacto do tratamento medicamentoso da endometriose nas questões profissionais, sexuais e econômicas das mulheres
}

Impact of endometriosis drug treatment on women's professional, sexual and economic issues Impacto del tratamiento farmacológico de la endometriosis en los problemas profesionales, sexuales y económicos de las mujeres

\section{RESUMO}

Objetivo: avaliar a melhora da qualidade de vida das mulheres com endometriose sob o tratamento medicamentoso e a demora no diagnóstico. Método: o estudo se deu por meio de inquérito on-line via plataforma GoogleForms ${ }^{\circledR}$, dirigido à mulheres diagnosticadas com endometriose. Resultado: foram entrevistadas um total de 476 mulheres (entre 19 e 38 anos de idade) residentes na cidade de Joinville e Região, a grande maioria nos estágios I e II de endometriose. A entrevista levantou dados sobre tempo até diagnóstico, medicamentos e terapêuticas utilizados, graus de comprometimento profissional, vida sexual e impacto econômico antes e após diagnostico e tratamento. Conclusão: o diagnóstico e tratamento correto para a endometriose, amenizando a sintomatologia e gastos extras, auxiliam na redução dos impactos sociais, econômicos e profissionais na vida das mulheres com essa doença.
\end{abstract}

DESCRITORES: Endometriose; Qualidade; Acesso e Avaliação da Assistência à Saúde; Saúde da Mulher.

\section{ABSTRACT}

Objective: evaluate the improvement in the quality of women's lives with endometriosis under drug treatment and the delay in diagnosis. Method: the study happened through an online survey via the GoogleForms ${ }^{\circledR}$ platform, aimed at women diagnosed with endometriosis. Result: a total of 476 women (between 19 and 38 years old) living in the city of Joinville and Region were interviewed, the vast majority in the stages I and II of endometriosis. The interview raised informations about the time since diagnosis, medications and therapeutic treatments used, levels of professional commitment, sexual life and economic impact before and after diagnosis and treatment. Conclusion: the diagnosis and correct treatment for endometriosis, which eases the symptoms and extra expenses, help to reduce the social, economic and professional impacts on the lives of women with this disease.

DESCRIPTORS: Endometriosis; Health Care Quality; Access and Evaluation; Women's health.

\section{RESUMEN}

Objetivo: evaluar la mejora en la calidad de vida de mujeres con endometriosis en tratamiento farmacológico y el retraso en el diagnóstico. Método: el estudio se realizó a través de una encuesta en línea a través de la plataforma GoogleForms ${ }^{\circledR}$, dirigido a mujeres diagnosticadas con endometriosis. Resultado: se entrevistó a un total de 476 mujeres (entre 19 y 38 años) residentes en la ciudad de Joinville y Región, la gran mayoría en las etapas I y II de la endometriosis. La entrevista recogió datos sobre tiempo hasta el diagnóstico, medicamentos y terapias utilizadas, grados de compromiso profesional, vida sexual e impacto económico antes y después del diagnóstico y tratamiento. Conclusión: el correcto diagnóstico y tratamiento de la endometriosis, aliviando los síntomas y los gastos extras, ayudan a reducir los impactos sociales, económicos y profesionales en la vida de las mujeres con esta enfermedad.

DESCRIPTORES: Endometriosis; Calidad; Acceso y Evaluación de la Atención de Salud; Salud de la Mujer.

RECEBIDO EM: 29/03/2021 APROVADO EM: 16/04/2021

\section{Caroline Trindade}

Graduandas em Medicina, Universidade da Região de Joinville, UNIVILLE.

ORCID: 0000-0002-5900-6613 
Marcia Francisca Moro Longo

Graduandas em Medicina, Universidade da Região de Joinville, UNIVILLE.

ORCID: 0000-0002-8410-1794

\section{Gabriela Santos Bianchin}

Graduandas em Medicina, Universidade da Região de Joinville, UNIVILLE.

ORCID: 0000-0001-8547-6937

\section{Laura Novochadlo Kluppel}

Graduandas em Medicina, Universidade da Região de Joinville, UNIVILLE.

ORCID: 0000-0002-6294-2840

\section{Hauana Heilig Martins}

Graduandas em Medicina, Universidade da Região de Joinville, UNIVILLE.

ORCID: 0000-0003-1003-037X

\section{Luciano Henrique Pinto}

Professor Adjunto, Departamentos de Medicina, Enfermagem e Farmácia, Universidade da Região de Joinville, UNIVILLE, Coordenador do Projeto Integrado ECOSAM.

ORCID: 0000-0003-0250-7502

\section{INTRODUÇÃO}

A endometriose é uma doença inflamatória provocada por células do endométrio que, ao invés de serem expelidas pelo canal vaginal, seguem um caminho conhecido como "menstruação retrógada", no qual saem pelas trompas e se aderem a tecidos de órgãos próximos onde se fixam e causam um processo inflamatório. É uma condição clinica que afeta 6,5 milhões de mulheres no Brasil e 176 milhões no mundo ${ }^{1}$.

Essa inflamação provocas fortes dores que se intensificam no período menstrual, levando a serem confundidas com cólicas menstruais severas. Essa dor intensa pode incapacitar as pacientes de exercerem suas atividades habituais, fator esse agravado pela demora - em média de 6 anos - em se ter o diagnóstico definitivo, visto que pode ser confundida com cólicas menstruais e o acesso aos exames são lentos no Sistema Único de Saúde. Dentre as características que afetam a qualidade de vida das mulheres, tem-se o incomodo durante as relações sexuais, sangramento intestinal e urinário e dificuldade em engravidar $40 \%$ das mulheres com endometriose são estéreis ${ }^{1}$.

A demora para se definir o diagnóstico implica em um longo período de espera que é acompanhada de não somente do-

\section{Dentre as}

características que

afetam a qualidade

de vida das

mulheres, tem-se o

incomodo durante

as relações sexuais,

sangramento

intestinal e urinário

e dificuldade em

engravidar - $40 \%$

das mulheres com

endometriose são

estéreis. res, mas também significativos impactos psicológicos e sociais para as mulheres, no qual a reversão de tais condições só ocorrem mediante a confirmação do diagnóstico e início do tratamento farmacológico indicado.

Dentre os principais impactos destaca-se a dificuldade de exercer uma profissão devido a necessidade de ausência por dores intensas, as dificuldades de ordem afetivas por dificuldade na realização do ato sexual, marcado por dores e falta de prazer devido a esta condição clínica; além da questão financeira para acesso a exames que confirmem a doença para início do tratamento, sendo muitos disponibilizados no SUS após longa lista de espera. Nesse cenários a dúvida que norteia essa pesquisa é qual a percepção de melhora que se obtém pelas mulheres, nos quesitos vida profissional, vida sexual e aspecto financeiro com o uso das medicações, frente ao não uso durante o período de espera do diagnóstico? Seriam essas positivas e em termos relativos ao tempo sem uso da medicação, demonstrando assim melhoras nos aspectos de vida profissional, sexual e financeira; visto que são as situações que mais afligem as mulheres com endometriose ${ }^{1,2}$.

$\mathrm{O}$ artigo tem como objetivo avaliar a melhora da qualidade de vida das mulheres com o tratamento medicamentoso e a demora no diagnóstico. Ademais irá abor- 
dar as dificuldades geradas para a paciente ao ser acometida pela doença e obter diagnóstico tardio. Não só os sintomas, mas também os tratamentos serão abordados no artigo para um entendimento completo da enfermidade.

\section{MÉTODO}

Este estudo se deu por meio de inquérito on-line feito a mulheres diagnostica- das com endometriose. Foi desenvolvido de maneira virtualizada via plataforma GoogleForms ${ }^{\oplus}$ em função da pandemia do COVID-19. Tratou-se de um levantamento com diversas perguntas a respeito de questões sobre demora no diagnóstico, opiniões sobre aspectos a qualidade de vida mais afetadas pela endometriose. Por envolver seres humanos, o presente projeto foi submetido ao Comitê de Ética em Pesquisa (CEP) da UNIVIL-
Figura 1: Processo de pesquisa, que incluiu questionário virtualizado disponibilizado para grupos de mulheres com endometriose em meios digitais (e-mail cadastrado na Farmácia de componente especializado).

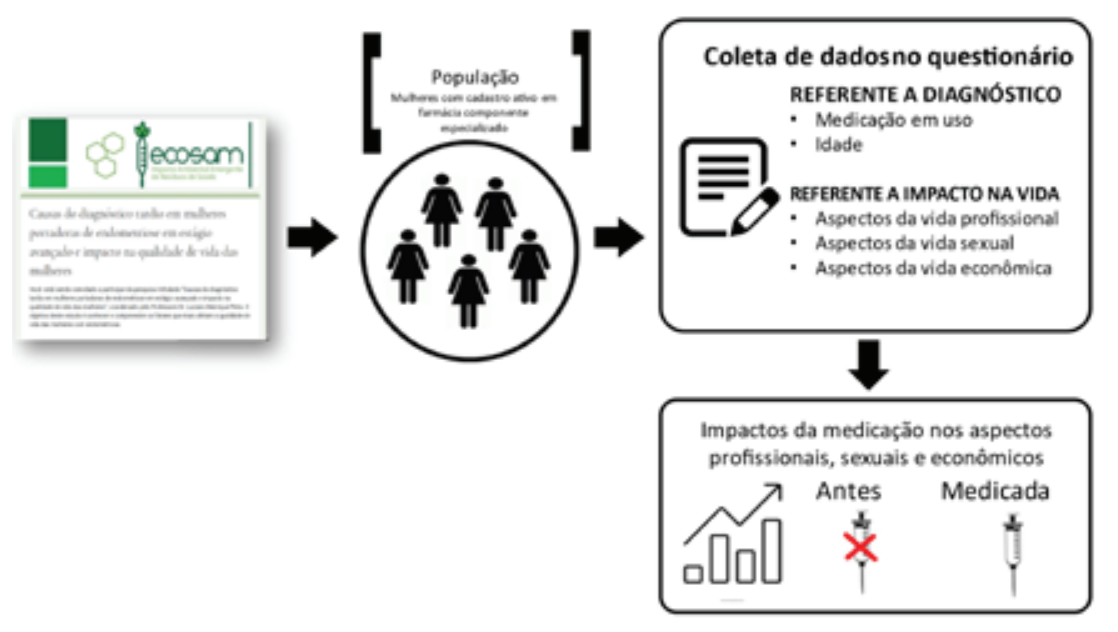

Fonte: Os pesquisadores

\section{Figura 2: Medicamentos e classes (fitoterápicos, anticoncepcionais,} analgésicos) utilizadas pelas pacientes na amostra em estudo.

\section{Medicações e/ou classes de medicamentos}

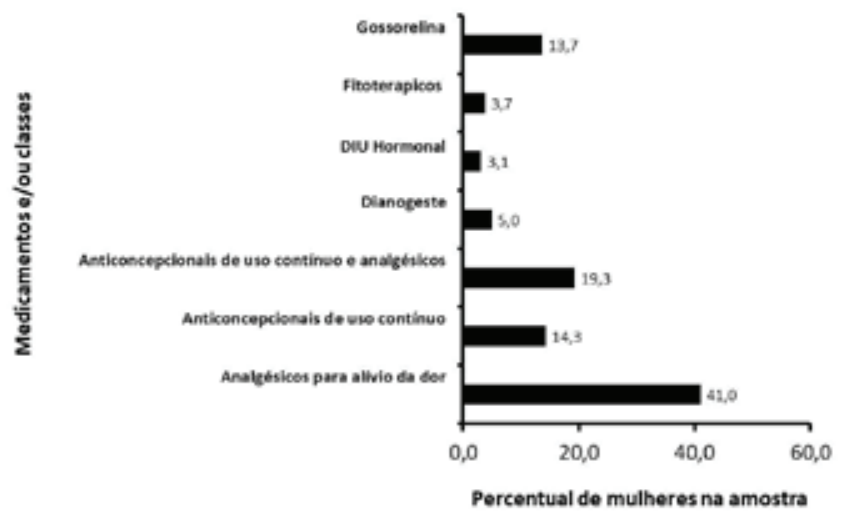

Fonte: Resultado da pesquisa
LE, sendo aprovado com registro CAA 26897619.2.0000.5366.

A população estudada correspondeu a mulheres - entre 18 a 35 anos - que relataram ter tido o diagnóstico de endometriose; cadastradas em no programa de dispensação de medicamentos excepcionais da cidade de Joiniville, que responderam o questionário via e-mail. Foram excluídos da pesquisa mulheres que não se enquadravam nestas condições, que não aceitaram participar da pesquisa ou que preencheram de forma incompleta o questionário. $\mathrm{O}$ estudo foi observacional, de análise documental e entrevistas,. Os dados e informações coletadas correspondiam a: [a]Características do paciente: idade, histórico familiar, hábitos gerais; [b]Aspectos do diagnóstico: tempo de demora, diagnósticos equivocados e [c] principais dificuldades: impacto na vida sexual, econômica e profissional (Figura 1). Foram excluídas do estudo questionários incompletos, com dados conflitantes e de mulheres sem diagnóstico confirmado de endometriose, avaliado pela descrição das mesmas sobre exames e consultas até o diagnóstico.

Os dados foram plotados em planilha do Excel $^{\circ}$ sendo agrupados conforme as variáveis de estudo levantadas anteriormente. Os dados plotados em planilha do Excel foram expressos em formas de gráficos para análise e discussão dos resultados.

\section{RESULTADOS E DISCUSSÃO}

Foram entrevistadas um total de 476 mulheres residentes na cidade de Joinville e Região. A idade variou de 19 anos até 38 anos, sendo a maioria (96\%) casada ou em relacionamento estável. A grande maioria se encontrava nos Estágios I e II da doença, com espera de diagnóstico de 6 anos contados a partir da primeira dor incapacitante. As medicações e classes principais de uso no momento da entrevista seguem listadas na Figura 2:

Os resultados demonstraram a presença de diferentes e diversos medicamentos e classes terapêuticas para o tratamento 
da endometriose e, principalmente, para alívio sintomático da dor pélvica. O número total de medicamentos e terapêuticas utilizadas foi de 586, alguns deles usados em associações, destacando-se a associação entre anticoncepcionais e analgésicos (Figura 2).

$\mathrm{O}$ tratamento da endometriose ocorre segundo a forma que a mesma se manifesta, existindo 4 estágios dessa doença ${ }^{3}$,

Figura 3: Escala de satisfação para vida profissional. Antes do tratamento $44.5 \%$ diziam ter comprometimento com a vida profissional por faltas ao trabalho e dificuldade de concentração. Pós-tratamento o grau de comprometimento caiu pra 29\%.

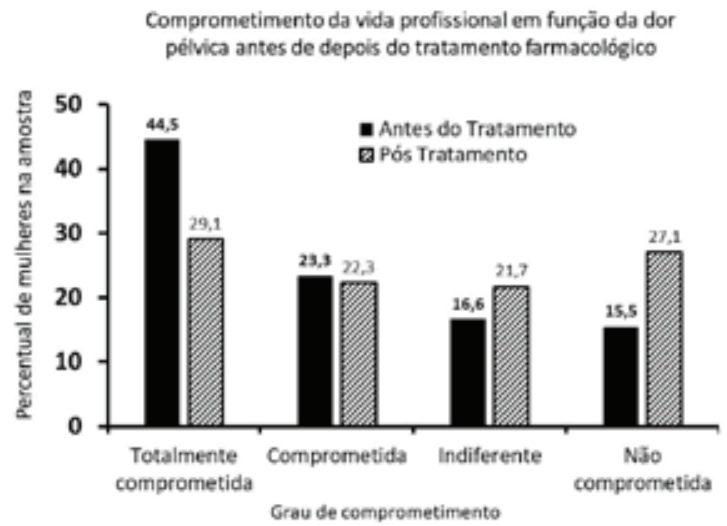

Fonte: Resultado da pesquisa

Figura 4: Escala de satisfação para vida sexual. Antes do tratamento $19 \%$ diziam ter satisfação com a vida sexual. Pós-tratamento o grau de comprometimento subiu para $23 \%$. A insatisfação caiu de $49.2 \%$ para $30,8 \%$.

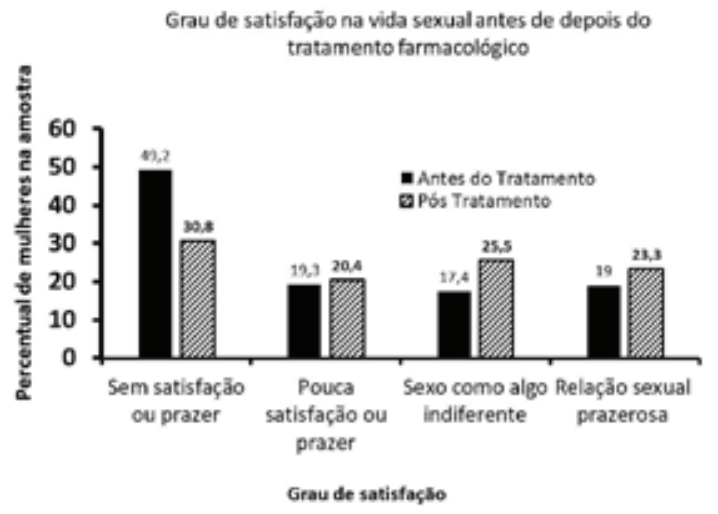

Fonte: Resultado da pesquisa do por se ter mais de 41 nódulos, no qual além do tratamento previsto em III, pode se ter também a retirada do úteró.

Entretanto, a demora até se ter o diagnóstico definitivo e o acesso a medicação correta acarreta impactos diversos na vida da mulher, sendo que um dos principais diz respeito a sua capacidade de realizar atividades profissionais quando em dor. Esta foi então uma variável incluída neste estudo, cujo resultados estão na figura 3:

As narrativas de mulheres que apresentam o quadro de endometriose revelam uma dor intensa, que afeta diretamente as relações de trabalho delas, no que tange a presença e concentração. A dor é descrita como excruciante, incapacitante, que imobiliza essas mulheres. Desse modo, muitas mulheres acabam faltando no trabalho pois não conseguem realizar suas atividades normalmente ${ }^{2}$. Assim, as pacientes com endometriose podem exibir uma diminuição na produtividade no trabalho, uma elevada taxa de absenteísmos, diminuição do ganho mensal e menor chance de promoção e evolução na carreira profissional ${ }^{5}$. Tal condição, sob o prisma do total comprometimento das atividades laborais, foi relatada por cerca de $44 \%$ das mulheres, com um decréscimo de $15 \%$ destas quando passaram a se tratar com a medicação. O grupo de mulheres que citou não haver comprometimento passou de $15,5 \%$ para $27,1 \%$ quando em tratamento, mostrando que a terapia medicamentosa de certa forma contribui para a melhoria das atividades de trabalho².

Além da dor, o aumento do fluxo menstrual, sintoma da doença, também causa grande incomodo. Há relatos de vergonha, medo e angústia relacionados à experiência de sujar-se com menstruação e sentirem-se expostas. Ou seja, além de conviverem constantemente com essa dor, essas mulheres também convivem com o medo da exposição social e a vergonha, sendo está questão também motivo de preocupação ${ }^{2,6}$.

No entanto, outro aspecto bastante observado nas mulheres com endometriose diz respeito a qualidade da vida sexual ${ }^{7}$. A dor no ato sexual, denominada dispa- 
reunia, é um dos sintomas mais descritos pelas doentes com endometriose. Além da dor de fato, o medo da própria afeta o desejo, a excitação e a resposta sexual humana pela elaboração cognitiva e emocional da dor. A doença também afeta o interesse e a atividade sexual, bem como a satisfação e prazer sexual prévios e a capacidade em atingir o orgasmo ${ }^{8}$. Na figura 4 temos o perfil do grupo de mulheres com suas respostas frente ao antes e após tratamento medicamentoso.

Dentro do tratamento medicamentoso, grande parte das mulheres relataram o uso de analgésicos e/ou anticoncepcionais. Os analgésicos, apesar de serem eficazes para aliviar a dor, são prejudiciais se usados em excesso, podendo causar a redução da libi$\mathrm{do}^{7,9}$. Os anticoncepcionais são bastante indicados para o tratamento da endometriose $^{10}$, são muito eficazes e poucos efeitos adversos são relatados, não prejudicando a libido. O dienogeste tem o mesmo efeito no tratamento da endometriose que as medicações anticoncepcionais ${ }^{11}$, mas pode haver perda de libido7. O DIU hormonal não altera libido. Por sua vez, danazol, e a gosserrelina podem diminuir a libido e causar diminuição da lubrificação"

Considerando os problemas vividos

figura 5: escala de percepção de gastos dentro do orçamento familiar. antes do tratamento $35 \%$ diziam ter gastado mais que o permitido e contado com ajuda de amigos e familiares. pós-tratamento o grau de comprometimento orçamentário caiu para $26,3 \%$.

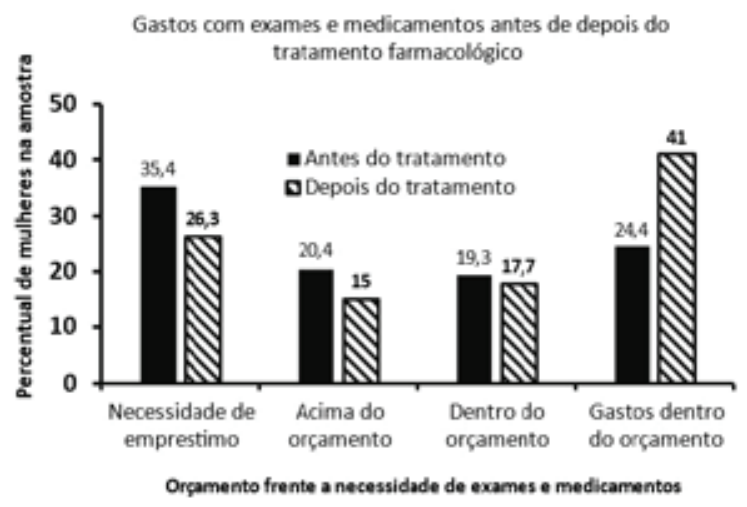

Fonte: Resultado da pesquisa

\section{Dentro do tratamento}

medicamentoso,

grande parte das

mulheres relataram o

uso de analgésicos e/

ou anticoncepcionais.

Os analgésicos, apesar

de serem eficazes

para aliviar a dor, são

prejudiciais se usados

em excesso, podendo

causar a redução da

libido. por essas mulheres, o tratamento medicamentoso se apresenta como uma boa alternativa, pois apresenta o potencial de melhorar não apenas a dor da mulher, mas também sua vida sexual ${ }^{12}$, indicando que a dispaurenia trata-se do fator que mais reflete na baixa satisfação do ato sexual, sendo a ocorrência de efeitos adversos sob a libido não tão influente.

Nota-se novamente a importância que o tratamento medicamentoso trás para a qualidade de vida da mulher, e que a questão da demora do diagnóstico se configura mais uma vez em um fator impactante para o seu bem-estar ${ }^{1}$.

Mas o diagnóstico ainda encontra problemas, no que diz respeito ao seu processo em si, que inclui a busca de exames e consultas por especialistas que não são tão rapidamente ofertados pelo SUS, o que de certo modo obriga a mulher a custear certos procedimentos de saúde, que também afeta seu orçamento, conforme mostra figura 5:

O gráfico da figura 5 demonstra os gastos das mulheres antes do início do tratamento, deixando evidente que 35,4\% dessas mulheres declaravam ter que recorrer a empréstimos para aquisição dos exames e medicamentos, contando ainda com ajuda de amigos e familiares para tais despesas. Situação essa que caiu cerca de $9 \%$ pós-tratamento, indicando que tal queda pode ser devido a não necessidade de custeio de exames. Ademais, 24,9\% das mulheres declararam que possuíam gastos dentro de seus orçamentos antes do início do tratamento.

No que corresponde ao número de mulheres no qual o tratamento se encaixava no orçamento, o número subiu de $24,9 \%$ para $41 \%$.

Esses dados de custos antes e após o início do tratamento medicamentoso demonstrou que, ao se fazer um diagnóstico correto, rápido e se iniciar o tratamento de forma adequada, os gastos desnecessários com grande número de exames e vários medicamentos para controle de sintomas e de dor diminuem consideravelmente ${ }^{13}$. Isso evidencia ainda mais a necessidade $\mathrm{da}$ conscientização da população para buscar 
atendimento profissional, além dos profissionais de saúde terem treinamento para conhecimento dos exames necessários e disponibilidade de tratamentos.

Mesmo a endometriose sendo uma doença impactante, o acesso aos serviços, ao diagnóstico e aos tratamentos é escasso $^{6,14}$. O que ocorre com essas mulheres, muitas das vezes, é a chamada violência institucional das instituições prestadoras de serviços públicos - como hospitais, postos de saúde e o judiciário - perpetuada por pessoas que deveriam garantir uma atenção humanizada, preventiva e, também, reparadora de danos ${ }^{14}$. Nos hospitais, a negligência e o descaso em relação aos usuários não são fora do comum, fazendo com que as necessidades básicas das pacientes não sejam atendidas, ou ainda, envolvendo procedimentos desnecessários e/ou indesejáveis ${ }^{15}$.

Dessa forma, as mulheres começam uma peregrinação por acesso a diagnóstico, tratamento e cuidados: elas esbarram em cenários hostis e com profissionais

\section{Demostrando}

assim, a grande

importância do

diagnostico ágil,

visando a melhora

na qualidade de vida

dessas mulheres a

partir dos efeitos

positivos gerados

pelo tratamento. diversos onde a violência comparece no atendimento rápido e pouco esclarecedor; na migração das mulheres por diferentes profissionais, inclusive nos planos de saúde; na desinformação/desconhecimento dos profissionais sobre a doença e seu manejo clínico; na escassez de acesso a serviços especializados; no alto custo das consultas particulares e tratamentos propostos.

\section{CONCLUSÃO}

A partir dos estudos realizados conclui-se portanto, que após as mulheres serem diagnosticadas com endometriose e serem tratadas corretamente com medicamentos como anticoncepcionais, analgésicos e fitoterápicos, os impactos sociais, econômicos e profissionais melhoram consideravelmente. Demostrando assim, a grande importância do diagnostico ágil, visando a melhora na qualidade de vida dessas mulheres a partir dos efeitos positivos gerados pelo tratamento.

\section{REFERÊNCIAS}

1. Harris $H R$, Wieser $F$, Vitonis $A F$, et al. Early life abuse and risk of endometriosis. Human Reproduction 2018; 33: 1657-1668.

2. Reis FM, Coutinho LM, Vannuccini S, et al. Is Stress a Cause or a Consequence of Endometriosis? Reprod Sci 2020; 27: 39-45.

3. Alkatout I, Wedel T, Maass N. Kombinierte Therapie der Endometriose: Radikal und schonend zugleich. Aktuel Urol 2018. 49: $60-72$

4. Findeklee S, Radosa JC, Hamza A, et al. Treatment algorithm for women with endometriosis in a certified Endometriosis Unit. Minerva Ginecol; 72. Epub ahead of print March 2020. DOI: 10.23736/S0026-4784.20.04490-1.

5. Falcone T, Flyckt R. Clinical Management of Endometriosis. Obstetrics \& Gynecology 2018; 131: 557-571.

6. Marinho MCP, Magalhaes TF, Fernandes LFC, et al. Quality of Life in Women with Endometriosis: An Integrative Review. Journal of Women's Health 2018; 27: 399-408.

7. La Rosa VL, De Franciscis P, Barra F, et al. Sexuality in women with endometriosis: a critical narrative review. Minerva Med 111. Epub ahead of print April 2020. DOI: 10.23736/S00264806.19.06299-2

8. Tanbo T, Fedorcsak P. Endometriosis-associated infertility: aspects of pathophysiological mechanisms and treatment options. Acta Obstet Gynecol Scand 2017; 96: 659-667.
9. Vercellini P, Buggio L, Berlanda N, et al. Estrogen-progestins and progestins for the management of endometriosis. Fertility and Sterility 2016; 106: 1552-1571.e2.

10. Pereira ACC, Pereira MMA, Vale PM, et al. Comparação entre contraceptivos hormonais combinados e progestágenos isolados na efetividade do tratamento da endometriose: uma revisão de literatura / Comparison between combined hormonal contraceptives and progestogens in the effectiveness of the treatment of endometriosis: a literature review. BJHR 2021; 4: 4081-4093.

11. Murji A, Biberoğlu K, Leng J, et al. Use of dienogest in endometriosis: a narrative literature review and expert commentary. Current Medical Research and Opinion 2020; 36: 895-907.

12. Ferrero S, Evangelisti G, Barra F. Current and emerging treatment options for endometriosis. Expert Opinion on Pharmacotherapy 2018; 19: 1109-1125.

13. Schwartz K, Llarena NC, Rehmer JM, et al. The role of pharmacotherapy in the treatment of endometriosis across the lifespan. Expert Opinion on Pharmacotherapy 2020; 21: 893-903.

14. São Bento PA de S, Moreira MCN. A experiência de adoecimento de mulheres com endometriose: narrativas sobre violência institucional. Ciênc saúde coletiva 2017; 22: 3023-3032.

15. Kernel Networks Inc. Endometriosis and Quality of Life Assessed by EHP 30. CMR. Epub ahead of print 11 June 2019. DOI: 10.31525/ct1-nct03981991. 